\title{
DOS NUEVAS ESPECIES EN OXYPETALUM Y MARSDENIA (ASCLEPIADACEAE) DE ARGENTINA
}

\author{
por SERGIO A. CACERES MORAL
}

\section{Summary}

Two new species of Asclepiadaceae from Argentina are described and illustrated: Oxypetalum fontellae Cáceres and Marsdenia tressensii Cáceres \& Morillo.

\section{Oxypetalum fontellae Cáceres nov.sp. ${ }^{2}$}

Fig. 1

Herba erecta, caule ex base ramificationibus $15-30 \mathrm{~cm}$ alta. Folia erecta, breviter petiolatis, lineare-lanceolatis usque ad $45 \mathrm{~mm}$ long. $x 7 \mathrm{~mm}$ lat. Inflorescentia extra-axillaris umbelliformis 10-15-flora. Calyx laciniae lineari, erectae, intus glabrae extus pubescentes. Corolla badia, tubo breviore, lobuli reflexi ca.7,5 mm long. $x$ 1,3-1,5 mm lat. intus glabri extus tenuiter pubescentes. Corona albida lobulis cuadrangularibus versus dimidium in duo lobulis partitis, apice partito; appendix stigmatalis subconicus in apice versus in dua filamenta divergentia partita. Retinaculum obovatum apice recurvo 0,45-0,50 mm long. x 0,15 mm lat., caudiculis ca. $0,15 \mathrm{~mm}$ long. $\times 0,10 \mathrm{~mm}$ lat. cum dente brevis et rotundo, pollinio oblongo 0,26 $\mathrm{mm}$ long. $\times 0,13 \mathrm{~mm}$ lat. Fruto ignoto.

Typus: ARGENTINA. Corrientes, Concepción, $11 \mathrm{Km} \mathrm{NW}$ de Santa Rosa, en pendiente hacia un estero, en pastizal, 15.XII.1977, S.G. Tressens \& al. 937. (Holotypus CTES).

Planta herbácea, erecta, ramificada desde la base, $15-30 \mathrm{~cm}$ alt. Hojas erguidas, lineal-lanceoladas, hasta $45 \mathrm{~mm}$ long. $\times 7 \mathrm{~mm}$ lat., margen revoluto, pubescentes en ambas caras, brevemente pecioladas. Inflorescencias extraxilares, alternas, en umbelas 10-15-floras, pedúnculo pubescente, hasta $45 \mathrm{~mm}$ long., pedicelos pubescentes, hasta $14 \mathrm{~mm}$ long.; cáliz 
de lacinias lineales, erguidas, 2,5 mm long. x 0,6 mm lat., pubescentes en la cara externa y glabras en la interna, con 1-2 glándulas basales internas; corola color castaño, tubo breve, ca. $1 \mathrm{~mm}$, lóbulos subulados, reflexos, ca. 7,5 mm long. x 1,3-1,5 $\mathrm{mm}$ lat., glabros en la cara interna y pubérulos en la externa; corona blanquecina, lóbulos cuadrangulares, libres entre sí, dilatados en la parte media en apéndices laterales, 1,5-1,7 mm long. x 11,5 mm lat., ápice búfido; ginostegio subsésil; apéndice estigmático subcónico con dos filamentos divergentes de $1 \mathrm{~mm}$ long.; anteras cuadrangulares, $0,6 \mathrm{~mm}$ long. x 0,6 mm lat., apéndice membranáceo, 0,7 mm long. x $0,7 \mathrm{~mm}$ lat., de ápice retuso; retináculo obovado, 0,45-0,50 mm long. $\mathrm{x}$ $0,15 \mathrm{~mm}$ lat., ápice agudo curvado hacia afuera, caudículas horizontales, $0,15 \mathrm{~mm}$ long. $\mathrm{x} 0,10 \mathrm{~mm}$ lat., diente incluso, corto y redondeado, y polinios elipsoides, 0,26 mm long. x 0,13 mm lat. Fruto desconocido.

El género Oxypetalum consta de un centenar de especies que habitan regiones tropicales, subtropicales y templadas de América. En Argentina el área de distribución abarca el norte y centro del país, teniendo como límite sur a la provincia de Buenos Aires.

Con la nueva entidad se eleva a 31 el número de especies de Argentina, de las cuales 11 son volubles y 20 erectas.

$O$. fontellae es afín a $O$. arenicola Hauman ex Lillo, coleccionada hasta el presente sólo en la provincia de Buenos Aires.

Los caracteres diferenciales de ambas especies están resumidos en la siguiente clave:

1. Hojas lineales no más anchas de $5 \mathrm{~mm}$; lóbulos de la corola $5 \mathrm{~mm}$ long. x 1,5 mm lat.; lóbulos de la corona bífidos con los extremos divergentes; retináculo oblongo-alargado, 0,60 mm long. x 0,14 mm lat., diente alargado y estrecho, polinios $0,45 \mathrm{~mm}$ long. $\mathrm{x} 0,17 \mathrm{~mm}$ lat.

O. arenicola

Fig. 1. Oxypetalum fontellae. A, planta; B, flor; C, lóbulo de la corona; D y E, translador de frente y de perfil (Holotipo). 


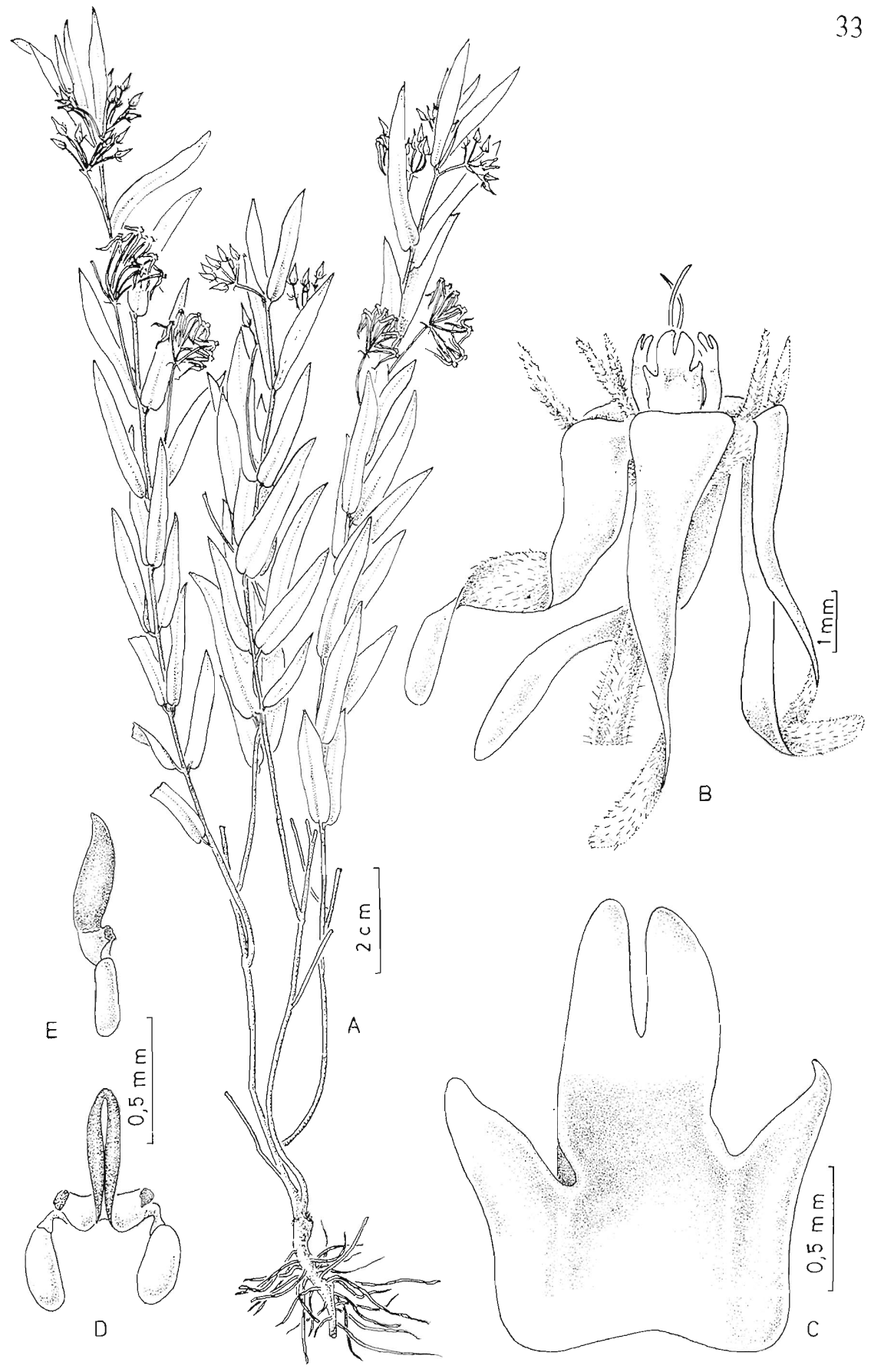


1. Hojas lineal-lanceoladas, generalmente más anchas de $5 \mathrm{~mm}$; lóbulos de la corola 7,5 mm long. x 1,5 mm lat.; lóbulos de la corona dilatados en la parte media en apéndices laterales, ápice bífido con los extremos no divergentes; retináculo obovado, 0,45-0,50 $\mathrm{mm}$ long. $\mathrm{x}$ $0,7 \mathrm{~mm}$ lat., diente corto y redondeado, polinios $0,26 \mathrm{~mm}$ long. $\mathrm{x} 0,13$ mm lat.

O. fontellae

Paratipos: Oxypetalum fontellae: ARGENTINA. Corrientes, Concepción, entre Santa Rosa y río Santa Lucía, 5.II.1968, Krapovickas 13777 (CTES); San Roque, ruta 17, $32 \mathrm{Km} \mathrm{SW}$ del desvío a Santa Rosa, en palmar de Butia yatay, suelo areno-arcilloso, 1.XII.1983, Tressens 2162 (CTES); Lavalle, ruta 12, $7 \mathrm{Km}$ de empalme con ruta 120, en campo bajo, planta erecta, 29-31.X.1974, Tressens 653 (CTES).

Oxypetalum arenicola: ARGENTINA. Buenos Aires, Rivera, F.C.O., 20.XII.1911, L. Hauman (Holotypus! BA).

\section{Marsdenia tressensii Cáceres \& Morillo ${ }^{3}$ nov.sp. ${ }^{4}$ Fig. 2}

Frutex volubilis; ramae juniores glabrae, vetusti notabiliter suberificati ac striati. Foliae ellipticae, subcoriaceae, apice abrupte vel tenuiter acuminato, basi acuta, 2-4 (6) glandulis in basi epiphylli. Inflorescentia umbelliforme pauciflora vel densiflora. Sepala ovata, ciliata. Corolla tubo urceolato, lobulis obovatis 5-6 mm long. x 3,3 mm lat., extus flavovirentibus intus eburneis. Coronae lobulis ovatotriangularibus, gynostegium adnatis ad stamines non superantibus. Gynostegio sessile. stigma conicum. Retinaculo lineari $0,40 \mathrm{~mm}$ long. $\times 0,06 \mathrm{~mm}$ lat., caudiculae 0,26 $\mathrm{mm}$ long., pollinio oblongo $0,83 \mathrm{~mm}$ long. $\times 0,23 \mathrm{~mm}$ lat.; foliculo subcylindrico atque $11 \mathrm{~cm}$ long. et $4 \mathrm{~cm}$ diam.; seminibus ovatis, compressis, margine integro.

Typus: ARGENTINA. Corrientes, Capital, Facultad de Ciencias Agrarias, cultivado a partir de una rama del ejemplar Cáceres 375, del río Aguapey y ruta 38,

3 Gilberto Morillo, Herbario Nacional de Venezuela, Jardín Botánico INPARQUES, Apartado 2146. Caracas.

4 Dedicamos esta especie a la Lic. S.G. Tressens quien la coleccionara por primera vez. 
pétalos verdoso-amarillentos por fuera, cara interna color crema, garganta con pelos blancos, mancha morado pálido en torno a la garganta y a lo largo de los lóbulos, flores muy fragantes, 21.X.1988, Cáceres 426. (Holotypus CTES), (Isotypi: ASU, BAA, BAB, C, FCQ, G, GH, HAS, K, LIL, LPB, MBM, MICH, MO, NY, P, SI, U, UB, VEN).

Trepadora, tallos jóvenes glabros, los más viejos marcadamente suberificados y estriados; láminas subcoriáceas, elípticas, hasta $17,5 \mathrm{~cm}$ long. x $7 \mathrm{~cm}$ lat., glabras, ápice abrupta a moderadamente acuminado, base aguda, 2-4 (6) glándulas en el epifilo; pecíolos 0,5-2,5 cm long., glabros; inflorescencia subaxilar, umbeliforme, 5-17-flora; pedúnculos 2-3(5) cm long., glabros; pedicelos 3-3,5 mm long., glabros; brácteas ovadas, 0,83 $\mathrm{mm}$ long. $\mathrm{x}$ 0,50 mm lat., ciliadas; sépalos ovados, 3,22 mm long. x 2,25 $\mathrm{mm}$ lat., ciliados, glabros interiormente, pubescentes en la cara externa, con una glándula en la base; corola con tubo urceolado, $6 \mathrm{~mm}$ long., verdoso, glabro por fuera, internamente con 5 bandas longitudinales de pelos blancos; lóbulos obovados, 5-6 mm long. x 3,3 mm lat, verdosoamarillentos por fuera, color crema por dentro, con mancha morado-pálido a lo largo de los mismos y en torno a la garganta, margen ralamente ciliado, revoluto; corona con lóbulos ovado-triangulares, 1,61 mm long. x 1,03 mm lat. en la base, adheridos totalmente al ginostegio y más cortos que las anteras; ginostegio sésil, 4,66 mm long., estigma cónico; anteras $1,16 \mathrm{~mm}$ long. $x$ 0,96 mm lat., membrana apical 1,42 $\mathrm{mm}$ long. $x$ 0,96 $\mathrm{mm}$ lat., cubriendo totalmente al estigma; retináculo lineal, $0,40 \mathrm{~mm}$ long. $\mathrm{x}$ 0,06 $\mathrm{mm}$ lat., caudículas $0,26 \mathrm{~mm}$ long., polinios oblongos, $0,83 \mathrm{~mm}$ long. x $0,23 \mathrm{~mm}$ lat.; fruto subcilíndrico hasta $11 \mathrm{~cm}$ long. $\mathrm{y}$ $4 \mathrm{~cm}$ diám., glabro, pericarpio grueso y duro; semillas ovadas, comprimidas, $6 \mathrm{~mm}$ long. x $3 \mathrm{~mm}$ lat., margen entero, espermopapus blanco, 1,5 $\mathrm{cm}$ long.

El género Marsdenia cuenta con aproximadamente 350 especies, de las cuales unas 120 son propias del continente americano.

En Argentina crecen en el NE $M$. macrophylla (H. et B.) Fourn. y $M$. tressensii Cáceres \& Morillo; y en el NW M. altissima (Jacq.) Dugand, $M$. castillonii Lillo ex Meyer, $M$. hassleriana Malme y M. ulei Schlch. et Rothe. 
Como resultado del análisis comparativo de las especies de la sección Ruehssia (Karst.) Fourn., a la cual pertenece $M$. tressensii, concluímos con el Dr. G. Morillo, que la misma es afín a M. hilariana Fourn.

M. hilariana habita en bosques húmedos desde 600-1500 m s.m., y se distribuye desde Colombia, Venezuela y Trinidad hasta el Distrito Federal y Mato Grosso en Brasil.

Ambas especies se diferencian por los siguientes caracteres:

1. Flores blanco-amarillentas; lóbulos de la corola oblongos, relación largo-ancho 2: 1, margen con muy pocas cilias irregularmente distribuidas; caudículas no ensanchadas en la unión con los polinios.

M. tressensii

1. Flores negras; lóbulos obovado-redondeados, relación largo-ancho 1:1, margen uniformemente ciliado; caudículas ensanchadas en la unión con los polinios.

M. hilariana

Para el recuento cromosómico se utilizaron raicillas, pretratadas con 8hidroxiquinoleína, fijadas en alcohol-acético 3:1, y coloreadas con orceína acética.

En metafase mitótica se puede apreciar que $M$. tressensiï posee $2 \mathrm{n}=22$. Este resultado coincide con los obtenidos para el género por Fritsch (1972); Ramakrishna \& Afzalunnisa (1981) y Navaneetham \& Sampath-kumar (1984).

Paratipos: $M$. tressensii: ARGENTINA. Corrientes, Ituzaingó, ruta 38 y río Aguapey, orilla río Aguapey, voluble; látex blanco, abundante; fruto verde, 7.I.1985, Tressens 3004 (CTES); río Aguapey y ruta 38, en selva en galería, voluble, 9.XII.1985, Cáceres 326 (CTES); Santo Tomé, ruta 38 y río Aguapey. en selva en galería, barranca arenosa, voluble, epifilo lustroso, 9.XII.1985, Cáceres 322 (CTES, VEN); ruta 38 y río Aguapey, en selva marginal, 24.II.1986, Cáceres 375 (CTES).

Fig. 2. Marsdenia tressensii. A, tallo adulto; B, rama joven; C, flor; D, corola abierta; E, corona y ginostegio; F, gineceo; $\mathrm{G}$, translador; $\mathrm{H}$, fruto; I, semilla; $\mathrm{J}$, cromosomas. (A - G, J, Holotipo; H - I. Tressens 3004). 


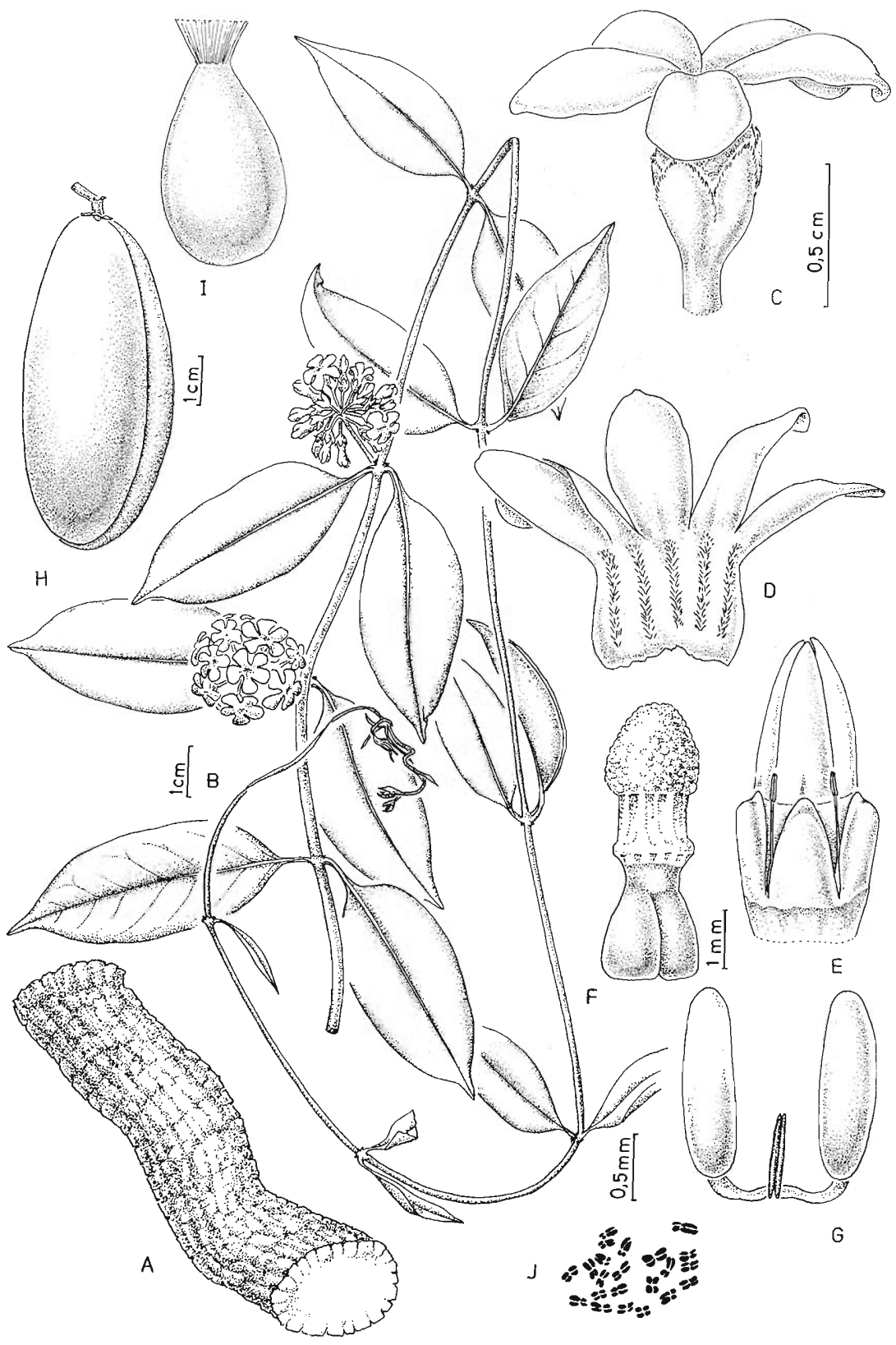


M hilariana: VENEZUELA. Yaracuy, Cerro Tigre, $10 \mathrm{~km}$ E of Aroa. Alt. 800-1000 m s.m.; flower black with white throat, 30.III.1980, Liesner y González 9711 (CTES, VEN).

Agradezco al Ing. Agr. A. Fernández las sugerencias en el estudio citológico.

\section{Bibliografía}

Arenas, P. \& G. C. Giberti. 1987. Datos etnobotánicos y morfológicos de Marsdenia castillonii Lillo ex Meyer (Asclepiadaceae) planta comestible del Gran Chaco. Candollea 42(1): 147-158.

Cáceres Moral, S.A. 1989. Nuevas citas de Asclepiadaceae para Argentina. Bonplandia 6(3): 173-182.

Fritsch, R. 1972. in Moore, R.J. 1977. Index to plant chromosome numbers for 1973-1974. Regnum Vegetabile 96: 28, 213.

Hoehne, F. C. 1916. Monographia das Asclepiadaceas Brasileiras. Oxypetalum R. Br. Comm. Linh. Telegr. Estrat. Matto-Grosso ao Amazonas 38(1): 1-131.

Meyer, T. 1943. Revisión de las especies argentinas del género Oxypetalum, Asclepiadaceae. Lilloa 9: 5-72.

- 1944. in Descole, Genera et Species Plantarum Argentinarum 2: 1-274. Morillo, G. 1974. Tres nuevas especies de Marsdenia. Acta Bot. Venez. 9(1-4): 311-315.

- 1974. El género Marsdenia en Venezuela, Colombia y Ecuador. Acta Bot. Venez. 13(1-4): 23-74.

Navaneetham, N. \& R. Sampathkumar, 1984. Chromosome number report LXXXII, Taxon 33:126-134.

Occhioni, P. 1956. Contribuiçào ao estudo do genero Oxypetalum. Arch. Jard. Bot. Rio de Janeiro 14: 37-210.

Ramakrishna, T. M. \& K. Afzalunnisa, 1981. Chromosome number report LXXIII, Taxon 30: 851-852.

Rambo, B. 1958. Die Gattung Oxypetalum in Rio Grande do Sul, Südbrasilien. Sellowia 9: 117-145.

Rothe, W. 1915. Ueber die Gattung Marsdenia R. Br. und die Stammpflanze der Condurangorinde. Bot. Jahrb. Syst. 52: 354-434. 